\title{
Editorial: Peer-to-peer networking and applications, volume 3, issue 2
}

\author{
Xuemin (Sherman) Shen • Heather Yu
}

Published online: 13 April 2010

(C) Springer Science+Business Media, LLC 2010

Six original research works are included in this issue of the Peer-to-Peer Networking and Applications. They address research problems related storage caching, peer-to-peer streaming, searching in KAD networks, security in multi-party $\mathrm{P} 2 \mathrm{P}$ computation, content prefetching, and gender difference in music downloading.

Computer and networking technologies are advancing very fast. Today's off the shelf processors are capable of executing multiple instructions in a single nano-second. Computer memory is getting faster and larger. High bandwidth and low latency communication technologies are capable of delivering high volume of data. Storage devices are also getting larger, but staying behind in terms of data delivery rate. As a result, for many data processing applications, the performance entirely depends on the throughput of these storage devices. A typical way to boost the performance of such applications is to use cache. In the first paper of this issue, "SPACE: A Lightweight Collaborative Caching for Clusters", Akon et al. propose a collaborative caching scheme for tightly coupled network workstations to enhance the performance of data driven applications.

In the second paper, "CliqueStream: Creating an Efficient and Resilient Transport Overlay for Peer-

\footnotetext{
X. Shen $(\bowtie)$

Department of ECE, University of Waterloo,

Waterloo, Ontario, Canada

e-mail: xshen@bbcr.uwaterloo.ca

H. Yu

Huawei Technologies, Bridgewater, NJ, USA

e-mail: heathery@ieee.org
}

to-Peer Live Streaming using a Clustered DHT", Asaduzzaman et al. address the problem of random selection of neighbors in overlay networks. Random neighbors results in longer distant traversal before data is delivered to the destination. This causes delay, which is very critical in live streaming. In this paper, authors propose to exploit locality properties of members of an overlay to overcome the problem.

The initial popularity of $\mathrm{P} 2 \mathrm{P}$ networks was due to their robustness in content distribution. To facilitate efficient search, often Distributed Hash Table (DHT), is used to organize contents. However, DHT enabled network suffers from information loss, partitions, and instability due to peer churns. In the third paper, "Evaluating and Improving the Content Access in KAD", Steiner et al. address this issue in KAD, an implementation of Kademlia. The paper identifies key design parameters of KAD and evaluates the impact of them using an analytical model. The paper also propose a scheme to improve the lookup latency.

In applications, such as collaborative filtering, distributed computation of trust and reputation, distributed monitoring, privacy of inputs to each node while performing a cooperative computation is crucial. In the paper, "Peer-to-Peer Secure Multi-Party Numerical Computation Facing Malicious Adversaries", Bickson et al. propose a bridge between existing distributed systems security theories and practical P2P deployment. In the paper, authors consider two security models-(1) system where peers correctly follow the protocol, but makes attempts to reveal private information, and (2) system where peers behave arbitrarily.

Many P2P applications require support for range queries, satisfying range constraints. In unstructured $\mathrm{P} 2 \mathrm{P}$ network, some form of flooding is employed to 
facilitate search. Replication of content reduces the cost of query processing and poorly replicated contents is a major source of query processing cost. In the fifth paper, "Popularity-aware Prefetch in P2P Range Caching”, Wang et al. propose a popularity-aware prefetching scheme to facilitate caching of poorlyreplicated contents those are potentially requested in subsequent range queries. Authors also show that with the proposed scheme, the overall query processing cost decreases significantly under various query load settings.

The impacts of $\mathrm{P} 2 \mathrm{P}$ computing in our social lives have started to attract attentions of researchers from diverse domains. In the last paper of this issue, "Gender Differences in Digital Music Distribution Methods", Tzantzara and Economides investigate different digital music distribution methods among Greek Internet users. Their empirical studies show that use of $\mathrm{P} 2 \mathrm{P}$ is the most common among music distribution methods. The authors expect that their findings will help music and advertising industries to specifically target their male and female customers.

In closing, we would like to acknowledge the contributions of the authors who submitted their works and the reviewers who provided their expert opinions and constructive and helpful comments. We would also like to extend our sincere thanks to Melissa Fearon, the Senior Editor, Emma Kalb, and Cristina Chua of Springer, for their support and help in bringing forward Volume 3, Issue 2 of this journal. We hope that you will enjoy the current issue and find the journal useful.

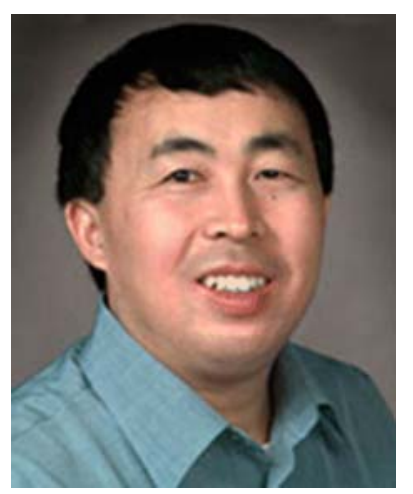

Xuemin (Sherman) Shen (IEEE M'97-SM'02-F'09) received the B.Sc. (1982) degree from Dalian Maritime University (China) and the M.Sc. (1987) and Ph.D. degrees (1990) from Rutgers University, New Jersey (USA), all in electrical engineering. He is a Professor and University Research Chair, Department of Electrical and Computer Engineering, University of Waterloo, Canada. Dr. Shen's research focuses on mobility and resource management in interconnected wireless/wired networks, UWB wireless communications networks, wireless network security, wireless body area networks and vehicular ad hoc and sensor networks. He is a co-author of three books, and has published more than 400 papers and book chapters in wireless communications and networks, control and filtering. Dr. Shen served as the Tutorial Chair for IEEE ICC'08, the Technical Program Committee Chair for IEEE Globecom'07, the General Co-Chair for Chinacom'07 and QShine'06, the Founding Chair for IEEE Communications Society Technical Committee on P2P Communications and Networking. He also serves as a Founding Area Editor for IEEE Transactions on Wireless Communications; Editor-in-Chief for Peer-to-Peer Networking and Application; Associate Editor for IEEE Transactions on Vehicular Technology; KICS/IEEE Journal of Communications and Networks, Computer Networks; ACM/Wireless Networks; and Wireless Communications and Mobile Computing (Wiley), etc. He has also served as Guest Editor for IEEE JSAC, IEEE Wireless Communications, IEEE Communications Magazine, and ACM Mobile Networks and Applications, etc. Dr. Shen received the Excellent Graduate Supervision Award in 2006, and the Outstanding Performance Award in 2004 and 2008 from the University of Waterloo, the Premier's Research Excellence Award (PREA) in 2003 from the Province of Ontario, Canada, and the Distinguished Performance Award in 2002 and 2007 from the Faculty of Engineering, University of Waterloo. Dr. Shen is a registered Professional Engineer of Ontario, Canada, and a Distinguished Lecturer of IEEE Communications Society.

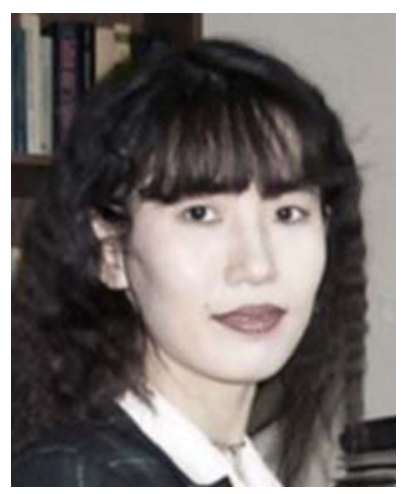

Heather Yu received my Ph.D. in Electrical Engineering from Princeton University in 1998. From September 1998 to March 2007, she was with Panasonic Princeton Laboratory where her major focus was multimedia communication and multimedia security R\&D. Currently she is the Senior Manager of Media Technologies at Huawei Technologies USA. One of her responsibilities is to lead the establishment of the multimedia content networking research team in New Jersey, USA. Her current research interests include human centric multimedia communications, IPTV, Peer-to-peer networking, mobile multimedia, etc. She is serving as the Associate Editor-in-Chief of the Journal of Peer-to-Peer Networking and Applications, Associate Editor of IEEE Multimedia Magazine and IEEE Communications Surveys and Tutorials, and Chair of the Human Centric Technical Subcommittee of IEEE ComSoc. She served as Technical Program Chair for several IEEE multimedia and communications conferences, the Chair of IEEE Multimedia Communications Technical Committee, and a voting member at ComSoc GITC, ComSoc SPC (strategic planning committee), etc. She has published 2 books, more than 60 technical papers and holds 23 US patents. 